\title{
The Fracture Analysis around Anti-plane Crack in An Infinite
}

\section{Piezoelectric Plate}

\section{Wenbin ZHAO ${ }^{a}$, Xuexia ZHANG ${ }^{b}$, Hailing XIE $^{c}$, Zhiyao ZHAO ${ }^{d}$}

School of Applied Science, Taiyuan University of Science and Technology, Taiyuan 030024, China

azwbhappy@aliyun.com, bxuexiaz@126.com, c903852334@qq.com, ${ }^{\text {a } 981786511 @ q q . c o m ~}$

Keywords: Infinite piezoelectric plate, Center crack, Complex function method, Mechanical strain energy release rate

Abstract. The fracture problem around the center crack in an infinite transversely isotropic piezoelectric plate under the anti-plane shear stress and the in-plane electric load at infinity is studied. Using the complex function method and undetermined coefficients method, the analytical solutions of the stress, displacement, electric displacement, electric potential, stress intensity factor, electric displacement intensity factor and the mechanical strain energy release rate around the crack tip are obtained with the assumption that the surface of the crack was electrically impermeable. The influence of the crack length, the mechanical load and the electric load on the mechanical strain energy release rate is analyzed with numerical analysis. The results show that the crack length and the mechanical load are always promoting the crack extension, the positive electric field promotes crack extension, and the negative electric field restrains it.

\section{Introduction}

Piezoelectric materials produce an electric field when stressed, and deform when subjected to an electric field. Such intrinsic coupling has attracted wide industrial applications of piezoelectric materials in various technologies, such as sensors, actuators, electromechanical transducers, medical instruments, sonar equipment, and so on. With increasingly wide applications of piezoelectric materials in engineering, the fracture problem in piezoelectric materials becomes extremely important, and has been studied by several researchers. Parton first analyzed a finite crack subjected to a far-field uniform tension by assuming the crack is mechanically traction free and electrically permeable ${ }^{1}$. Using the Green function method, Deeg, Sosa, Pak and Chen studied the plane and anti-plane fracture problems for infinite piezoelectric materials by assuming the electrical condition of the crack is impermeable ${ }^{2-5}$. Park, Jelitto and Gao et al studied the crack growth criterion of piezoelectric material6 $6^{6-8}$. It was shown that the electric loading may promote or retard crack propagation. The energy release rate as the crack growth criterion was in contradiction to experimental observations. Zhao, Zhang, Tong and Gao studied an elliptical cylinder cavity and the electric field within the cavity in a piezoelectric materia by the conformal mapping method ${ }^{9-12}$. Wang, Liu, Zhang and Jaroon analyzed intensity factor around the crack embedded in an infinite piezoelectric material under a far-field anti-plane mechanical load and a far-field in-plane electrical load using permeable boundary condition ${ }^{13-16}$. Using the complex variable function method and the technique of the conformal mapping, Xue, Lu and Guo discussed the fracture problem of two semi-infinite collinear cracks in a piezoelectric strip under the anti-plane shear stress and in-plane electric load on the partial crack surface ${ }^{17-19}$. The stress intensity factors (SIFs) of interface crack in a 
piezoelectric bi-material is found by a combination of the Stroh formalism and the Williams eigenfunction method ${ }^{20-22}$.

Using the complex function methods and undetermined coefficients methods, the paper studies the fracture problem around the anti-plane crack in an infinite transversely isotropic piezoelectric plate. Under the anti-plane shear stress and the in-plane electric load at infinity, the mechanical problem of anti-plane crack tip field can be reduced to a set of partial differential equation boundary value problem. By solving partial differential equation boundary value problem, the stress, displacement, electric displacement, electric potential, stress intensity factor, electric displacement intensity factor and the mechanical strain energy release rate around the crack tip are derived. At last, the influence of the crack length, the mechanical load and the electric load on the mechanical strain energy release rate is analyzed with numerical analysis.

\section{The fundamental equations}

Consider a transversely isotropic piezoelectric body in the form of an infinite plate containing a center crack subjected to mechanical load $\sigma_{0}$ and electrical load $D_{0}$ as shown in Figure 1 . The crack is located at the center, and the length is $2 a$. In a fixed rectangular coordinate system $x_{j}(j=1,2,3), x_{1} o x_{2}$ plane is transversely isotropic plane, $x_{3}$ axis is perpendicular to the $x_{1} o x_{2}$ plane.

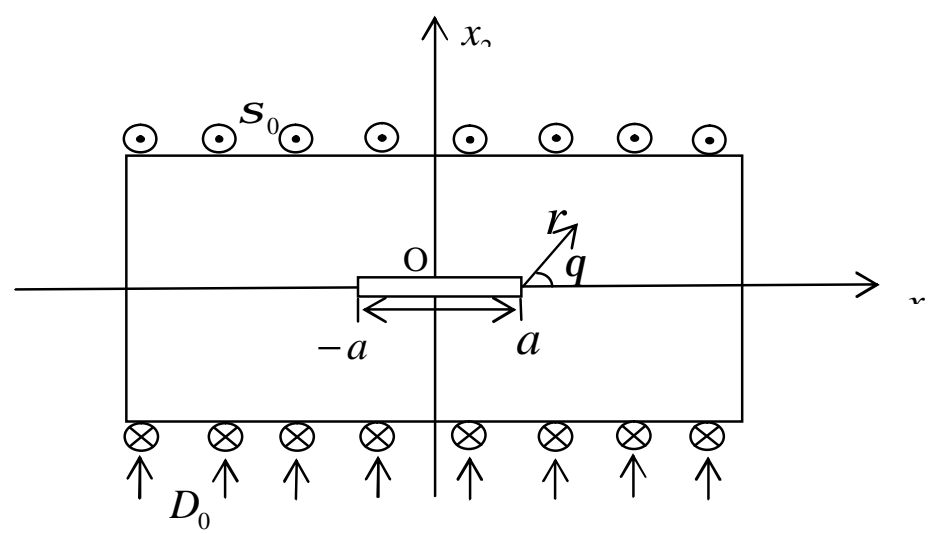

Fig. 1 Infinite piezoelectric plate with a center

Here we only address general two-dimensional problems in the plane, all variables are constant along the $x_{3}$-axis. The piezoelectric boundary value problem is simplified considerably if we consider only the out-of-plane displacement and the in-plane electric fields in the forms:

$$
u_{1}=u_{2}=0, u_{3}=u_{3}\left(x_{1}, x_{2}\right), E_{1}=E_{1}\left(x_{1}, x_{2}\right), E_{2}=E_{2}\left(x_{1}, x_{2}\right), E_{3}=0
$$

Where $u_{i}$ and $E_{i}$ are the elastic displacements and electric field, respectively.

In the absence of body forces and free charge, the equilibrium equations are simplified to

$$
c_{44} \nabla^{2} u_{3}+e_{15} \nabla^{2} \phi=0, \quad e_{15} \nabla^{2} u_{3}-\varepsilon_{11} \nabla^{2} \phi=0
$$

Where $\nabla^{2}$ is the two-dimensional Laplacian operator in the variables $x_{1}$ and $x_{2}$. 
For general piezoelectric materials, the elastic constants $c_{44}$, piezoelectric constants $e_{15}$ and dielectric constants $\varepsilon_{11}$ satisfy $c_{44} \varepsilon_{11}+e_{15}{ }^{2} \neq 0$. So, the equilibrium equation (2) is simplified into two independent harmonic equations as follows:

$$
\nabla^{2} u_{3}=0, \quad \nabla^{2} \phi=0
$$

These equations are to be solved subject to the boundary conditions

$$
\begin{aligned}
& x_{2} \rightarrow \infty: \sigma_{32}=\sigma_{0}, \quad D_{2}=D_{0} \\
& x_{2}=0,\left|x_{1}\right|<a: \sigma_{32}=0, D_{2}^{+}=D_{2}^{-}=0
\end{aligned}
$$

Therefore, an analysis of fracture problem near cracks tip for an infinite transversely isotropic piezoelectric plate subjected to the mechanical load $\sigma_{0}$ and electrical load $D_{0}$ can be reduced to finding the solution of the boundary value problem of partial differential equation (3-5).

\section{Undetermined coefficients method}

From Complex function theory, it can be found that the real part and the imaginary part of the analytic function of complex variable $z_{1}=x_{1}+i x_{2}$ are the solutions of the harmonic equations (3). The following functions can be chosen as the elastic displacement and electric potential containing two real undetermined coefficients, respectively.

Letting

$$
u_{3}=a_{1} \operatorname{Re}\left(\overline{U_{1}}\right)+b_{1} \operatorname{Im}\left(\overline{U_{1}}\right), \phi=a_{2} \operatorname{Re}\left(\overline{U_{2}}\right)+b_{2} \operatorname{Im}\left(\overline{U_{2}}\right)
$$

Where $a_{j}, b_{j}(j=1,2)$ are the real undetermined coefficients, $U_{1}, U_{2}$ are the stress function and electric potential function. Then the elastic stresses and electric displacements can be expressed through the stress function and electric potential function.

In order to solve the boundary value problem of partial differential equation (3-5), noticing the anti-plane shear stress $\sigma_{0}$ and the in-plane electric load $D_{0}$, the stress function and electric potential function are selected as follows ${ }^{9}$

$$
U_{1}=\frac{\sigma_{0} z_{1}}{\left(z_{1}^{2}-a^{2}\right)^{\frac{1}{2}}}, U_{2}=\frac{D_{0} z_{1}}{\left(z_{1}^{2}-a^{2}\right)^{\frac{1}{2}}}
$$

When $x_{2} \rightarrow \infty, \quad U_{1}=\sigma_{0}, \quad U_{2}=D_{0}$

When $x_{2}=0,\left|x_{1}\right|<a, U_{1}=-i \frac{\sigma_{0} x_{1}}{\left(a^{2}-x_{1}^{2}\right)^{\frac{1}{2}}}, \quad U_{2}=-i \frac{D_{0} x_{1}}{\left(a^{2}-x_{1}^{2}\right)^{\frac{1}{2}}}$

With the help of the boundary conditions, we can obtain a system of non-homogeneous linear equations in 4 unknowns about coefficients $a_{j}, b_{j}(j=1,2)$. 


$$
c_{44} b_{1} \sigma_{0}+e_{15} b_{2} D_{0}=\sigma_{0}, e_{15} b_{1} \sigma_{0}-\varepsilon_{11} b_{2} D_{0}=D_{0}
$$

$(10)$

$$
c_{44} a_{1} \frac{\sigma_{0} x_{1}}{\left(a^{2}-x_{1}^{2}\right)^{\frac{1}{2}}}+e_{15} a_{2} \frac{D_{0} x_{1}}{\left(a^{2}-x_{1}^{2}\right)^{\frac{1}{2}}}=0, \quad e_{15} a_{1} \frac{\sigma_{0} x_{1}}{\left(a^{2}-x_{1}^{2}\right)^{\frac{1}{2}}}-\varepsilon_{11} a_{2} \frac{D_{0} x_{1}}{\left(a^{2}-x_{1}^{2}\right)^{\frac{1}{2}}}=0
$$

Solving non-homogeneous linear equations, the unique solution of equations can be derived :

$$
a_{1}=0, a_{2}=0, \quad b_{1}=\frac{\sigma_{0} \varepsilon_{11}+D_{0} e_{15}}{\left(c_{44} \varepsilon_{11}+e_{15}{ }^{2}\right) \sigma_{0}}, \quad b_{2}=\frac{e_{15} \sigma_{0}-c_{44} D_{0}}{\left(c_{44} \varepsilon_{11}+e_{15}{ }^{2}\right) D_{0}}
$$

Substituting (12) into (6), the real analytic solutions are obtained, which meet the equilibrium equation (3) and the boundary conditions $(4,5)$.

\section{Stress intensity factor and electric displacement intensity factor}

When $z_{1} \rightarrow a, \quad U_{1} \rightarrow \frac{\sigma_{0} a}{\left[2 a\left(z_{1}-a\right)\right]^{\frac{1}{2}}}, \quad U_{2} \rightarrow \frac{D_{0} a}{\left[2 a\left(z_{1}-a\right)\right]^{\frac{1}{2}}}$

Fully considering the loadings, we introduce the following stress intensity factor and electric displacement intensity factor

$$
K^{\sigma}=\lim _{z_{1} \rightarrow a}\left[2\left(z_{1}-a\right)\right]^{\frac{1}{2}} U_{1}=\sigma_{0} \sqrt{a}, K^{D}=\lim _{z_{1} \rightarrow a}\left[2\left(z_{1}-a\right)\right]^{\frac{1}{2}} U_{2}=D_{0} \sqrt{a}
$$

Thus, near the crack tip, it can be seen that

$$
U_{1}=\frac{K^{\sigma}}{\left[2\left(z_{1}-a\right)\right]^{\frac{1}{2}}}, \quad \text { as } z_{1} \rightarrow a ; \quad U_{2}=\frac{K^{D}}{\left[2\left(z_{1}-a\right)\right]^{\frac{1}{2}}}, \quad \text { as } z_{1} \rightarrow a
$$

Using (12) and (15), the stress expressions and electric displacement expressions near the mode crack $\left(z_{1} \rightarrow a\right)$ of the infinite transversely isotropic piezoelectric material can be written as

$$
\begin{aligned}
& \sigma_{31}=\frac{\left(c_{44} \varepsilon_{11} \sigma_{0} D_{0}+c_{44} e_{15} D_{0}{ }^{2}\right) K^{\sigma}+\left(e_{15}{ }^{2} \sigma_{0}{ }^{2}-c_{44} e_{15} \sigma_{0} D_{0}\right) K^{D}}{(2 r)^{\frac{1}{2}}\left(c_{44} \varepsilon_{11}+e_{15}{ }^{2}\right) \sigma_{0} D_{0}} \operatorname{Im}\left[\frac{1}{(\cos \theta+i \sin \theta)^{\frac{1}{2}}}\right] \\
& \sigma_{32}=\frac{\left(c_{44} \varepsilon_{11} \sigma_{0} D_{0}+c_{44} e_{15} D_{0}{ }^{2}\right) K^{\sigma}+\left(e_{15}{ }^{2} \sigma_{0}{ }^{2}-c_{44} e_{15} \sigma_{0} D_{0}\right) K^{D}}{(2 r)^{\frac{1}{2}}\left(c_{44} \varepsilon_{11}+e_{15}{ }^{2}\right) \sigma_{0} D_{0}} \operatorname{Re}\left[\frac{1}{(\cos \theta+i \sin \theta)^{\frac{1}{2}}}\right] \\
& D_{1}=\frac{\left(e_{15} \varepsilon_{11} \sigma_{0} D_{0}+e_{15}{ }^{2} D_{0}{ }^{2}\right) K^{\sigma}-\left(e_{15} \varepsilon_{11} \sigma_{0}{ }^{2}-c_{44} \varepsilon_{11} \sigma_{0} D_{0}\right) K^{D}}{(2 r)^{\frac{1}{2}}\left(c_{44} \varepsilon_{11}+e_{15}{ }^{2}\right) \sigma_{0} D_{0}} \operatorname{Im}\left[\frac{1}{(\cos \theta+i \sin \theta)^{\frac{1}{2}}}\right] \\
& D_{2}=\frac{\left(e_{15} \varepsilon_{11} \sigma_{0} D_{0}+e_{15}{ }^{2} D_{0}{ }^{2}\right) K^{\sigma}-\left(e_{15} \varepsilon_{11} \sigma_{0}{ }^{2}-c_{44} \varepsilon_{11} \sigma_{0} D_{0}\right) K^{D}}{(2 r)^{\frac{1}{2}}\left(c_{44} \varepsilon_{11}+e_{15}{ }^{2}\right) \sigma_{0} D_{0}} \operatorname{Re}\left[\frac{1}{(\cos \theta+i \sin \theta)^{\frac{1}{2}}}\right]
\end{aligned}
$$

where $r$ is polar radius and $\theta$ is polar angle, as shown in figure 1. 
From constitutive equation (2), letting $k=1$, we can obtain as follow:

$$
\frac{\partial u_{3}}{\partial x_{1}}=\frac{\varepsilon_{11} \sigma_{31}+e_{15} D_{1}}{c_{44} \varepsilon_{11}+e_{15}{ }^{2}}, \frac{\partial \phi}{\partial x_{1}}=\frac{e_{15} \sigma_{31}-c_{44} D_{1}}{c_{44} \varepsilon_{11}+e_{15}{ }^{2}}
$$

The polar coordinate expressions of displacement and electric potential can be written as

$$
u_{3}=\frac{\left(\varepsilon_{11} \sigma_{0}+e_{15} D_{0}\right) \cdot(2 r)^{\frac{1}{2}}}{\left(c_{44} \varepsilon_{11}+e_{15}{ }^{2}\right) \sigma_{0}} \cdot K^{\sigma} \cdot \operatorname{Im}\left[(\cos \theta+i \sin \theta)^{\frac{1}{2}}\right], \varphi=\frac{\left(e_{15} \sigma_{0}-c_{44} D_{0}\right) \cdot(2 r)^{\frac{1}{2}}}{\left(c_{44} \varepsilon_{11}+e_{15}{ }^{2}\right) D_{0}} \cdot K^{D} \cdot \operatorname{Im}\left[(\cos \theta+i \sin \theta)^{\frac{1}{2}}\right]
$$

\section{The mechanical strain energy release rate}

The mechanical strain energy release rate is chosen as the fracture criterion to analyze the fracture behavior at the crack tip in this paper. Let $K^{S}$ denote the strain intensity factor at the crack tip. By constitutive equation (2), $K^{S}$ can be written as

$$
K^{S}=\frac{e_{15} K^{D}+\varepsilon_{11} K^{\sigma}}{e_{15}{ }^{2}+c_{44} \varepsilon_{11}}=\frac{\sqrt{a}\left(\varepsilon_{11} \sigma_{0}+e_{15} D_{0}\right)}{c_{44} \varepsilon_{11}+e_{15}{ }^{2}}
$$

The mechanical strain energy release rate [10] can be expressed as

$$
G_{m}=\frac{K^{\sigma} K^{S}}{2}=\frac{\left(\varepsilon_{11} \sigma_{0}+e_{15} D_{0}\right) \sigma_{0} a}{2\left(c_{44} \varepsilon_{11}+e_{15}{ }^{2}\right)}
$$

To analyze the influence of the crack length $2 a$ and the mechanical/electric loads on the mechanical strain energy release rate, we take PZT-5H as model materials with the following material constants in table 1.

TABLE 1: THE MATERIAL PARAMETERS OF PIEZOELECTRIC CERAMICS

\begin{tabular}{ccccc}
\hline Materials & $c_{44} /\left(\times 10^{10} \mathrm{~N} / \mathrm{m}^{2}\right)$ & $e_{15} /\left(\mathrm{C} / \mathrm{m}^{2}\right)$ & $\varepsilon_{11} /\left(\times 10^{-10} \mathrm{C} / \mathrm{Vm}\right)$ & $G_{c r} /\left(\mathrm{J} / \mathrm{m}^{2}\right)$ \\
\hline PZT-5H & 3.53 & 17.0 & 151.0 & 5.0 \\
\hline
\end{tabular}

Figure 2 shows the influence of the crack length $2 a$ on the mechanical strain energy release rate. It can be seen that the mechanical strain energy release rate increases linearly with the increases of the crack length. This shows that the influence of the crack length on the mechanical strain energy release rate is significant. In addition, the slope of straight line increase with the increases of the mechanical loads $\sigma_{0}$, which indicates that the bigger the mechanical loads $\sigma_{0}$ increases, the faster the mechanical strain energy release rate increases. Similarly, the positive electric field has greater effects on the mechanical strain energy release rate than the negative electric field. Figure 3 shows the influence of the mechanical load $\sigma_{0}$ on the mechanical strain energy release rate. The mechanical strain energy release rate increases as the mechanical load $\sigma_{0}$ increases, which indicates that the mechanical loads $\sigma_{0}$ can easily lead to crack propagation. It can be found from Fig. 3 that the longer the crack length is, the bigger the mechanical strain energy release rate change. Moreover, the positive electric field has greater effects on the mechanical strain energy release rate than the negative electric field. The effect of the electric loads $D_{0}$ on the mechanical strain energy release rate is shown in figure 4 . The mechanical strain energy release rate is not only dependent on the size of the electric load $D_{0}$, and also related with the direction of the electric load $D_{0}$. The mechanical strain energy release rate increases with increasing the positive electric loads $D_{0}$, and increases with decreasing the negative electric loads $D_{0}$. It can be seen that the bigger the mechanical loads $\sigma_{0}$ and the longer the crack length, the greater the influence of the electric loads $D_{0}$ on the mechanical strain energy release rate. 


\section{Conclusions}

Based on the complex variable function method and the method of undetermined coefficients, the fracture problem of an infinite transversely isotropic piezoelectric plate is studied under the anti-plane shear stress and the in-plane electric load at infinity along the $x_{2}$ axis. The analytic solutions of the stress, displacement, electric displacement, electric potential, stress intensity factor, electric

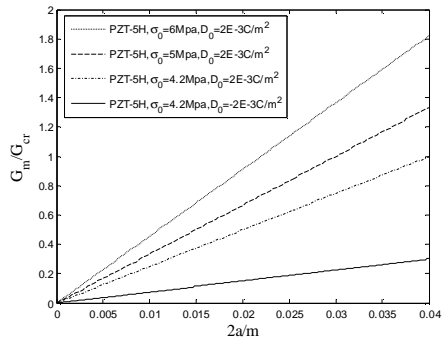

Fig. 2 the variation of $\mathrm{Gm} / \mathrm{Gcr}$ with of $\mathrm{Gm} / \mathrm{Gcr}$ with crack length

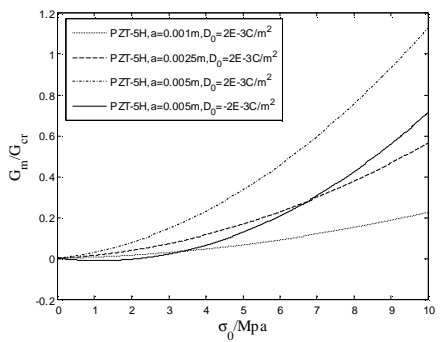

Fig.3 The variation of $\mathrm{Gm} / \mathrm{Gcr}$ with mechanical load $\sigma_{0}$

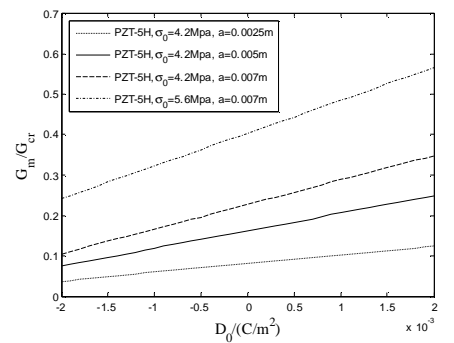

Fig. 4 The variation electric $\operatorname{load}_{D_{0}}$

displacement intensity factor and the mechanical strain energy release rate at the crack tip are obtained. For the given material PZT-5H, the variation curves of the mechanical strain energy release rate withthe crack length, the mechanical load $\sigma_{0}$ and the electric load $D_{0}$ are obtained by using the numerical calculation. The results show that the longer crack length and the bigger mechanical load lead to the greater of the mechanical strain energy release rate. The crack length and the mechanical load $\sigma_{0}$ are always promote the crack extension. The mechanical strain energy release rate is related to the size and direction of the electric field. The positive electric field promotes crack extension, and the negative electric field restrains it.

\section{Acknowledgments}

This work was financially supported by the National Natural Science Foundation of China (No. 51574171), the Natural Science Foundation of Shanxi Province (No. 201601D102003) and the Graduate Scientific and Technological Innovation Project of the Taiyuan University of Science and Technology (20151037).

\section{References}

[1] V. Z. Parton: Acta Astronautics Vol. 3 (1976), p. 671.

[2] W. F. J. Deeg: Ph. D. Thesis, Stanford University, 1980.

[3] H. A. Sosa and Y. E. Pak: International Journal of Solids and Structures Vol. 26 (1990), p. 1.

[4] Y. E. Pak: International Journal of Fracture Vol. 54 (1992), p. 79.

[5] Z. T. Chen and S. W. Yu: International Journal of Fracture Vol. 84 (1997), p. 25.

[6] S. B. Park and C. T. Sun: International Journal of Fracture Vol. 70 (1995), p. 203.

[7] H. Jelitto, F. Felten, M. V. Swain: Schneider: Applied Mechanics Vol. 74 (2007), p. 1197.

[8] H. Gao, T. Y. Zhang and P. Tong: J. the Mechanics and Physics of Solids Vol. 45 (1997), p. 491.

[9] W. B. Zhao, Z. X. Hu, X. X. Zhang: Theo. and Appl. Frac. Mech. Vol. 74 (2014), p. 1.

[10] T. Y. Zhang, C. F. Gao: Theoretical and applied fracture mechanics Vol. 41 (2004), p.339.

[11] R. M. McMeeking and A. Ricoeur: Inter. J. of Soli. and Struc. Vol. 40 (2003), p.6143.

[12] C. F. Gao, P. Tong and T. Y. Zhang: Comp. Sci. Tech. Vol. 65 (2005), p.1354.

[13] B. L. Wang, J. C. Han and S. Y. Du: Acta Mechanica Solida Sinica Vol. 25 (2004), p.399. 
[14] S. H. Liu, Z. G. Zhang and Z. Z. Zou: Engineering Mechanics Vol. 20 (2003), p.14.

[15] R. Jaroon and E. M. Mark: International Journal of Fracture Vol. 51 (2008), p.1.

[16] Z. G. Zhou and Z. T. Chen: Archive of Applied Mechanics Vol. 81 (2011), p.641.

[17] C. Xue, Y. H. Zhou and H. D. Yong: Acta Mechanica Solida Sinica Vol. 33 (2012), p.449.

[18] J. H. Guo, P. Liu and Z. X. Lu: Applied Mathematics and Mechanics Vol. 32 (2011), p.72.

[19] X.X. Zhang, X. C. Cui, W. Y. Yang: Appl. Math. Mech. Vol. 30 (2009), p.1489.

[20] O.Viun, A. Komarov, Y. Lapusta: Engineering Fracture Mechanics Vol. 15 (2018), p.143.

[21] M. Abe, T. Ikeda, M. Koganemaru: Engineering Fracture Mechanics Vol. 171 (2017), p.1.

[22] M. H. Zhao, H. T. Liu, G. T. Xu, Engineering Fracture Mechanics Vol. 153 (2016), p.289. 